\title{
UV laser-induced poling inhibition produces bulk domains in MgO-doped lithium niobate crystals
}

\author{
Andreas Boes, ${ }^{1,2, a}$ Hendrik Steigerwald, ${ }^{1,2}$ Didit Yudistira, ${ }^{1}$ Vijay Sivan, ${ }^{1,2}$ Scott Wade, ${ }^{3}$ \\ Sakellaris Mailis, ${ }^{4}$ Elisabeth Soergel, ${ }^{5}$ Arnan Mitchel1 ${ }^{1,2}$ \\ ${ }^{1}$ School of Electrical and Computer Engineering, RMIT University, Melbourne, VIC 3001, Australia \\ ${ }^{2}$ ARC Center for Ultra-high Bandwidth Devices for Optical Systems (CUDOS), RMIT University, \\ Melbourne, VIC 3001, Australia \\ ${ }^{3}$ Faculty of Science, Engineering and Technology, Swinburne University of Technology, Hawthorn, VIC \\ 3122, Australia \\ ${ }^{4}$ Optoelectronics Research Centre, University of Southampton, Highfield, Southampton, SO17 1BJ, United \\ Kingdom \\ ${ }^{5}$ Institute of Physics, University of Bonn, Wegelerstr. 8, 53115 Bonn, Germany
}

\begin{abstract}
We report the realization of high-resolution bulk domains achieved using a shallow, ferroelectric, domain inverted surface template obtained by UV laser-induced poling inhibition (PI) in MgO-doped lithium niobate. The properties of the obtained bulk domains are compared to those of the template and their application for second harmonic generation is demonstrated. The present method enables domain structures with a period length as small as $3 \mu \mathrm{m}$ to be achieved. Furthermore, we propose a potential physical mechanism that leads to the transformation of the surface template into bulk domains.
\end{abstract}

\footnotetext{
${ }^{a}$ Electronic mail: $\underline{\text { 3363819@student.rmit.edu.au }}$
} 
Lithium niobate $\left(\mathrm{LiNbO}_{3}\right)$ crystals have been widely used in many optical applications, in particular nonlinear optics, due to its excellent nonlinear optical properties [1]. Nonlinear optical processes such as frequency conversion are based on quasi-phase matching, which requires periodic domain inversion of the crystal's spontaneous polarization $P_{\mathrm{s}}$ at submicron-to-few microns periods depending on the wavelengths involved in the nonlinear process [2]. As it is the case for many nonlinear processes, frequency conversion also requires high intensity laser light, which may induce optical damage to the crystal that results in the distortion of the optical beam. It is known that adequate magnesium oxide $(\mathrm{MgO})$ doping in $\mathrm{LiNbO}_{3}$ can significantly reduced the optical damage [3]. However, while it is possible to find crystals suitable for high optical intensity applications, fine period domain inversion of $\mathrm{MgO}$-doped crystals is known to be particularly challenging due to random domain growth and a high leakage currents [4].

A common method used for domain patterning of $\mathrm{LiNbO}_{3}$ is electric field poling (EFP) [5], where a spatially modulated electric field is applied along the polar axis of the crystal. Domain inversion occurs in areas where the local electric field exceeds the coercive field $\left(E_{\mathrm{c}}\right)$. While this method has been shown to be an effective way to achieve domain patterning in congruent $\mathrm{LiNbO}_{3}$, it is not always successful when applied to MgO-doped $\mathrm{LiNbO}_{3}$, due to a pronounced sideways domain spreading which occurs during EFP limiting the smallest achievable domain period [5]. The ability to realize short domain periods on such crystals can widen the scope of its applications, as they are required for the generation of counter propagating photons in nonlinear optical applications [6]. Recently, it has been shown that a two-step voltage application during 
UV illumination can produce good quality domain patterns in $\mathrm{MgO}$-doped $\mathrm{LiNbO}_{3}$ with a period of $19 \mu \mathrm{m}[7,8]$. This technique first generates surface domains by applying an electric field, which is smaller than the $E_{\mathrm{c}}$ while simultaneously illuminating the crystal with weakly absorbed UV light. The weakly absorbed UV light reduces the $E_{\mathrm{c}}$ [9] therefore allowing the nucleation of surface domains. By applying a second EFP step without UV light illumination, the surface domains behave as a template, and bulk domains are created. The major drawback of using this technique especially when realizing small domains is the need to simultaneously irradiate the crystal with UV light during EFP. Especially, as the experimental setup required for EFP can potentially limit the focal spot size of the UV laser light on the crystal surface and thus affecting the attainable minimum period lengths of the domain pattern. Separating the two steps (UV illumination and electric field application) could overcome this limitation.

In this paper, we report on the fabrication of bulk domain structures in MgO-doped $\mathrm{LiNbO}_{3}$, which are pre-defined by a spatial modulation of the lithium (Li) concentration induced by strongly absorbed UV laser light irradiation. The bulk domain distribution is subsequently produced by an even number of EFP steps. In this way the laser irradiation and EFP steps can be separated removing the restrictions imposed in Refs. $[7,8]$. We report the fabrication of periodic bulk domain structures with a period as short as $3 \mu \mathrm{m}$.

The methodology for generating and visualizing the domains is presented below. In our experiments we used $500-\mu \mathrm{m}$-thick $5 \mathrm{~mol} \%$ of $\mathrm{MgO}$ doped $\mathrm{Z}$-cut congruent $\mathrm{LiNbO}_{3}$ crystals $(\mathrm{MgCLN})$, which were provided by 'Yamaju Ceramics co. Ltd.'. 
Focused UV laser light from a frequency doubled argon ion laser $(\lambda=244 \mathrm{~nm})$ was scanned along the crystallographic $\mathrm{Y}$ direction on the $+\mathrm{Z}$-face of the crystal. The laser beam was focused using a fused silica lens $(f=40 \mathrm{~mm})$ to a focal beam diameter of $\sim 6 \mu \mathrm{m}$ and an intensity of $\sim 3 \times 10^{5} \mathrm{~W} / \mathrm{cm}^{2}$. The laser beam was scanned to irradiate linear track sections with a period of $18.8 \mu \mathrm{m}$ at a velocity of $0.5 \mathrm{~mm} / \mathrm{s}$.

Local irradiation of the $+Z$-face with strongly absorbed UV laser light results in a Li deficiency enabling surface domain formation via poling inhibition (PI) during a subsequent EFP step $[9,10]$. For the EFP process we used a setup similar to the one introduced by Sones et al. [11], which allowed us to visualize the domain growth during the poling process. The voltage applied to the crystal was ramped up at a rate of $5 \mathrm{~V} /(\mathrm{mm} \mathrm{s})$ until a non-ohmic behavior of the measured current was observed indicating domain reversal [12]. After the completion of the poling process, the applied voltage was ramped down at a rate of $100 \mathrm{~V} /(\mathrm{mm} \mathrm{s})$. To avoid unintended domain inversion on specific parts of the crystal, areas of the crystal surface were covered with Kapton tape after each poling step. This step of process allowed us to study the domain formation after each poling step, namely after the first forward poling, after the first reverse poling (completing the first poling cycle), after the second forward poling and after the second reverse poling (completing the second poling cycle). The resulting domain patterns were investigated using scanning electron microscopy (SEM) and optical microscopy imaging after domain selective etching of the polished crystal cross-sections (Y-face) using hydrofluoric acid (HF).

An investigation to explore the smallest achievable domain period with this method was also conducted. For this purpose, we used a fused silica lens with a shorter 
focal length $(f=10 \mathrm{~mm})$ to achieve a focal beam diameter of $\sim 2 \mu \mathrm{m}$. The focused laser beam was then scanned in periodic track patterns (similar to above) with periods of $4 \mu \mathrm{m}$ and $3 \mu \mathrm{m}$ at a velocity of $0.5 \mathrm{~mm} / \mathrm{s}$. As previously described, the same methodology was adopted for the generation and visualization of domains.

The quality and thermal stability (at working temperatures) of the periodic domain structure after the application of two poling cycles was investigated by performing a second harmonic generation ( $\mathrm{SHG}$ ) experiment. A beam from a tunable infrared laser was focused into a heated, periodically poled crystal $(L=9.4 \mathrm{~mm}$, $\Lambda=18.8 \mu \mathrm{m}$ ) and the SHG power was detected as a function of the fundamental wavelength. The SHG power was monitored over a time period of two hours at crystal temperatures of $100,150,200$ and $260^{\circ} \mathrm{C}$ to provide an in-situ measure of the thermal stability of the domains.

The results of the UV irradiation and subsequent EFP are shown in Fig. 1. Fig. 1a shows the SEM image of the crystal after the first forward poling step, where a surface domain can be recognized from the ridge that is formed on the Z-face of the crystal and the differentially etched pattern of the Y-face cross-section. This shows that the polarization of the whole crystal is reversed except a shallow area underneath the UV-irradiated track, which maintains the original polarization of the crystal (PI domain). The Y-face cross-section also reveals that the tail-to-tail boundary of the PI domain is very irregular (spiky). The measured width of the surface domain is $\sim 5 \mu \mathrm{m}$, which is comparable to the focal diameter of the irradiating UV-laser beam. The measured depth of the PI domain is $\sim 1.5 \mu \mathrm{m}$. Higher UV laser light intensities were found to generate 
wider and deeper PI domains, however this were found to come at cost of thermal induced surface damage such as cracks. After a subsequent reverse poling step the volume underneath the PI domain maintains its polarization while domain reversal occurs everywhere else as shown in Fig. 1b. As a result, a well-defined bulk domain is formed below the PI domain. From the visualization of the domain growth during reverse poling, which is not shown here, we observed that the domain nucleation started within the UV irradiated area.

The domain depth is qualitatively analyzed on the cross-section using optical microscope imaging after HF treatment. The images of the sample taken after the first and the second poling cycle are shown in Figs. $2 a$ and 2b, respectively. The needle-like periodic structures in Fig. 2 correspond to the UV laser irradiation as shown previously in Fig. 1b. We find that the domain width is getting narrower as the crystal depth is increased and they do not terminate the opposite -Z-face of the crystal, which is unlike the poling process with standard EFP. As shown in Fig. 2a, we also find that after the first poling cycle the depth of the individual domains varies dramatically and their alignment does not nicely follow the crystallographic Z-axis. This however is improved after applying the second poling cycle as shown in Fig. 2b. Much deeper domain are achieved, which, in addition, are now more uniformly aligned along the Z-axis. The improvement of the domain uniformity in depth and parallelism after the second poling cycle can be attributed to a smoother and more regular domain wall motion, achieved when poling a crystal multiple times [13]. However, we observed that applying further poling cycles did not improve the quality of the domain in terms of the domain depth. The importance of the domain wall motion can be further supported by fact that this domain engineering 
method was not suitable for congruent $\mathrm{LiNbO}_{3}$, where the domain wall motion is faster compared to $\mathrm{MgO}-$ doped $\mathrm{LiNbO}_{3}[14]$.

In Figs. $3 \mathrm{a}$ and $3 \mathrm{~b}$ we show fabricated samples with smaller domain periods, namely $4 \mu \mathrm{m}$ and $3 \mu \mathrm{m}$, achieved using a lens with smaller focal length. We can see that the domain periods match nicely with the scanning periods of the beam and that the left domain in Fig. 3a is smaller than the other domains in the SEM images. These results demonstrate that the present technique is also applicable to obtain small poling period. However, good uniformity of the periodic domain structure over large areas was found to be difficult to achieve, which requires further investigation but it is beyond the scope of this paper. A smoother and slower domain wall motion, which could be beneficial for reaching this goal, might be achievable by using higher crystal temperatures during EFP [15] or by conditioning the crystal by subjecting it to repetitive poling cycles prior to UV irradiation and the final poling steps $[13,16]$.

The measured SHG power as a function of the fundamental pump wavelength of a sample at $100,150,200$ and $260^{\circ} \mathrm{C}$ is show in Fig. 4. The SHG powers follow approximately a sinc-function, which is an indication of overall good domain uniformity. The maximum of the curves are at a wavelength of $1537.95,1545.55,1553.95$ and $1565.8 \mathrm{~nm}$ for the corresponding crystal temperatures. The theoretical prediction for the temperatures that correspond to the measured maxima of the sinc-curves are 99, 151, 203 and $268^{\circ} \mathrm{C}$, when using the temperature dependent Sellmeier equation from Ref [17], which is in good agreement with the chosen temperatures. The spectral bandwidth of the curves is $\sim 1.25 \mathrm{~nm}$, which is close to the expected theoretical spectral bandwidth of 
$\sim 1.2 \mathrm{~nm}$. The difference in the spectral bandwidth may be due to the variations in the duty cycle or the variations of the domain width itself [18]. The graph also shows that the sinc-functions are not symmetrical, which is most apparent from the side-lobes. This may originate from a variation of the temperature profile along the sample. The monitored SHG power did not diminish over a time period of 2 hours for all the temperatures, indicating that the domains are stable in this temperature range, which makes these poling patterns suitable for high power applications.

A phenomenological model that describes the formation mechanism of the bulk domains that have been observed in our experiments is schematically illustrated in Fig. 5. The wavelength of the focused UV laser light is $244 \mathrm{~nm}$, which is well below the band edge of CLN, (around $350 \mathrm{~nm}$ [19]). Thus, the UV light is strongly absorbed, propagating only tens of nanometers into the surface of the MgCLN crystal before being dissipated [19], creating a localized heat profile with temperatures up to $\sim 1000^{\circ} \mathrm{C}$ [20] (Fig. 5a). The Li ions are mobile at this temperature and diffuse into the adjacent cooler part of the crystal [10], resulting in Li deficient region below the irradiated tracks and a Li enriched region around it (Fig. 5b). As the coercive field $E_{\mathrm{c}}$ depends on the Li concentration of the crystal [10], it can be expected that the $E_{\mathrm{c}}$ would increase in the Li deficient region while a decreased $E_{\mathrm{c}}$ is expected at the Li enriched region (Fig. 5c). When the forward poling step is applied (Fig. 5d), domains nucleate preferentially at the surface of the Li enriched regions next to the UV-irradiated tracks due to the reduced $E_{\mathrm{c}}$ [10] (Fig. 5e). Under the influence of the applied field, the nucleated domains grow into the depth of the crystal while spreading laterally to merge with its adjacent nucleated domains. However, the $\mathrm{Li}$ deficient area at the surface is poling inhibited (due to the its increased $E_{\mathrm{c}}$ ), and thus the 
domain inversion will not spread into these Li deficient regions resulting in a surface domain remaining just beneath the UV irradiated region at the end of the EFP step. This is illustrated schematically in Fig. $5 \mathrm{f}$ and corresponds to the observations in Fig. 1a.

When the reverse poling step is applied (illustrated in Fig. 5g), the domains nucleate again at the Li enriched area next to the irradiated UV tracks (Fig. 5h), spreading laterally and merging with its adjacent nucleated domains. However, directly underneath the poling inhibited regions, the crystal polarization does not invert, as was experimentally found (Fig. 1b and 2) and illustrated in Fig. 5i. This poling behavior can be explained by considering the surface domains acting as local electrical insulating barriers (similar to the properties of photoresist in standard EFP). The insulating nature of the surface domains is given, because they are already oriented along the applied electric field, therefore the internal polarization switching current cannot reach the crystal surfaces and be compensated by an external current. This is similar to the observation that a uniform surface domain layer, which is formed when fabricating titanium indiffused waveguides on $\mathrm{Z}$-cut $\mathrm{LiNbO}_{3}$, can prevent electric field poling of the crystal $[21,22]$. Furthermore, the peaks of the spiky shaped domain boundary might be pinned by defects in the Li deficient area and therefore they are even more resistive to domain inversion. Since the surface domains act as insulating barriers and the top domain wall is pinned in the Li deficient area of the PI domain, the polarization of the crystals below the surface domains prefers to have the same polarization orientation as the pinned domain wall and therefore generating the observed bulk domain, which propagates in a manner that is consistent with normal EFP. 
In summary, a method for the production of bulk domains based on the UV laser pre-irradiation of the $+Z$ polar surface of $\mathrm{MgCLN}$ crystals followed by a sequence of EFP steps have been presented. This method does not require simultaneous irradiation and application of an electric field thus enabling the fabrication of fine periodic domain structures with periods as short as $3 \mu \mathrm{m}$. A model is proposed suggesting that the bulk domains are generated because the PI surface domains, which are produced in the first poling step, act as electrical insulating barriers in subsequent poling steps. It was also determined that the uniformity in depth and parallelism of the bulk domains improved after a second full poling cycle. Although the top domain wall of the bulk domains might be pinned at defect sites, which may be influenced by elevated crystal temperatures, it was found that the bulk domains are stable at elevated temperatures while the good overall quality of the bulk domain distribution was verified by SHG experiments.

The authors acknowledge the facilities, and the scientific and technical assistance, of the Australian Microscopy \& Microanalysis Research Facility at RMIT University and the Faculty of Science, Engineering and Technology at Swinburne University of Technology. This work was made possible through the generous support of the ARC Centre of Excellence CUDOS.

\section{References:}

[1] L. Arizmendi, Phys. Stat. Sol. (a) 201, 253 (2004). 
[2] L.E. Myers, R.C. Eckardt, M.M. Fejer, R.L. Byer, W.R. Bosenberg, and J.W. Pierce, J. Opt. Soc. Am. B 12, 2102 (1995).

[3] D.A. Bryan, R. Gerson, and H.E. Tomaschke, Appl. Phys. Lett. 44, 847 (1984).

[4] K. Mizuuchi, A. Morikawa, T. Sugita, and K. Yamamoto, J. Appl. Phys. 96, 6585 (2004).

[5] M. Yamada, N. Nada, M. Saitoh, and K. Watanabe, Appl. Phys. Lett. 62, 435 (1993).

[6] Canalias and V. Pasiskevicius, Nature Photonics 1, 459 (2007).

[7] E. Kitado, M. Fujimura, and T. Suhara, Appl. Phys. Express 6, 102204 (2013).

[8] M. Fujimura, E. Kitado, T. Inoue, and T. Suhara, Photonics Technology Letters, IEEE 23, 1313 (2011).

[9] C.L. Sones, A.C. Muir, Y.J. Ying, S. Mailis, R.W. Eason, T. Jungk, Á. Hoffmann, and E. Soergel, Appl. Phys. Lett. 92, 072905 (2008).

[10] H. Steigerwald, M. Lilienblum, F. von Cube, Y. Ying, R. Eason, S. Mailis, B. Sturman, E. Soergel, and K. Buse, Phys Rev B 82, 214105 (2010).

[11] C.L. Sones, M.C. Wengler, C.E. Valdivia, S. Mailis, R.W. Eason, and K. Buse, Appl. Phys. Lett. 86, 212901 (2005).

[12] M.C. Wengler, M. M ller, E. Soergel, and K. Buse, Appl. Phys. B 76, 393 (2003).

[13] V. Gopalan, T.E. Mitchell, and K.E. Sicakfus, Solid State Communications 109, 111 (1998).

[14] K. Nakamura, J. Kurz, K. Parameswaran, and M.M. Fejer, J. Appl. Phys. 91, 4528 (2002).

[15] H. Ishizuki, I. Shoji, and T. Taira, Appl. Phys. Lett. 82, 4062 (2003).

[16] C.Y.J. Ying, G.J. Daniell, H. Steigerwald, E. Soergel, and S. Mailis, J. Appl. Phys. 114, 083101 (2013).

[17] O. Gayer, Z. Sacks, E. Galun, and A. Arie, Appl. Phys. B 91, 343 (2008).

[18] M.M. Fejer, G.A. Magel, D.H. Jundt, and R.L. Byer, Quantum Electronics, IEEE Journal of 28, 2631 (1992).

[19] A.M. Mamedov, M.A. Osman, and L.C. Hajieva, Applied Physics A 34, 189 (1984). 
[20] A.C. Muir, G.J. Daniell, C.P. Please, I.T. Wellington, S. Mailis, and R.W. Eason, Applied Physics A 83, 389 (2006).

[21] J. Amin, V. Pruneri, J. Webjorn, P.S.J. Russell, D.C. Hanna, and J.S. Wilkinson, Opt. Commun. 135, 41 (1997).

[22] D. Janner, D. Tulli, F. Lucchi, P. Vergani, S. Giurgola, and V. Pruneri, Appl. Phys. A 91, 319 (2008).
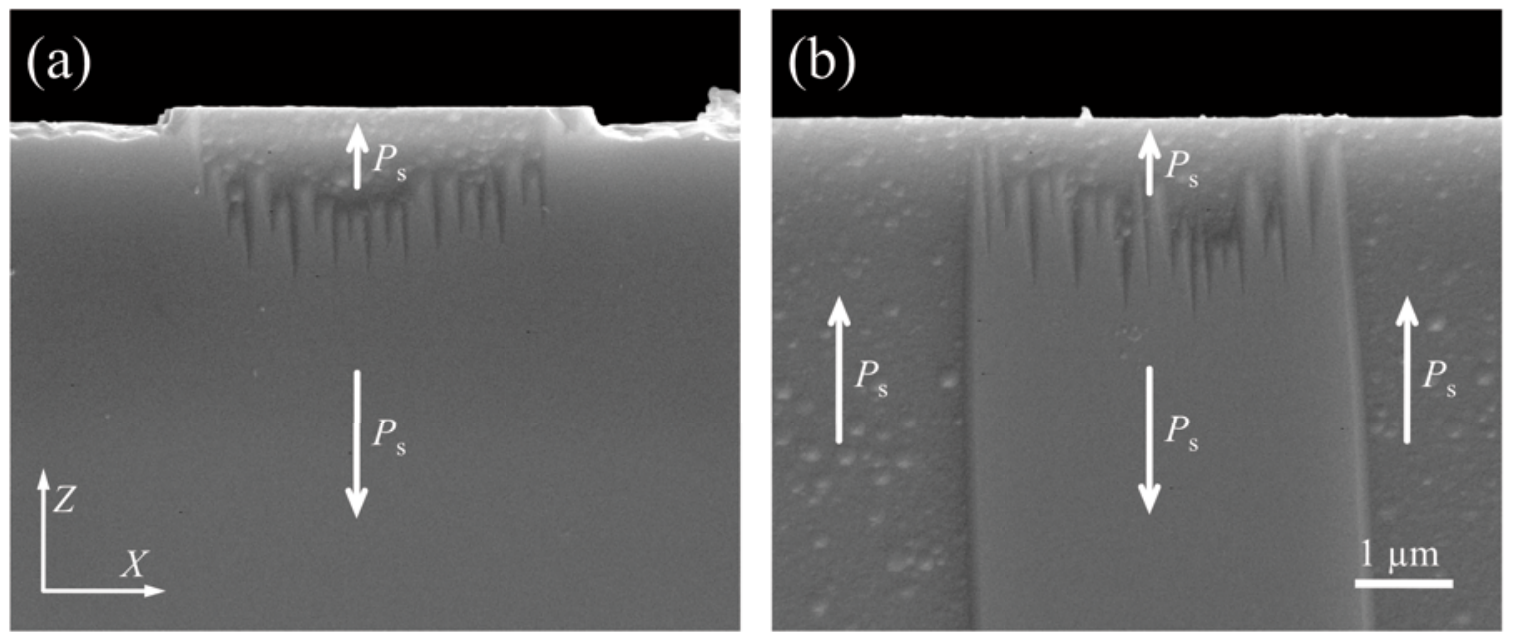

FIG. 1. SEM images of the sample crossed-sections after being treated with HF, taken after (a) forward poling and (b) reverse poling of the crystal.
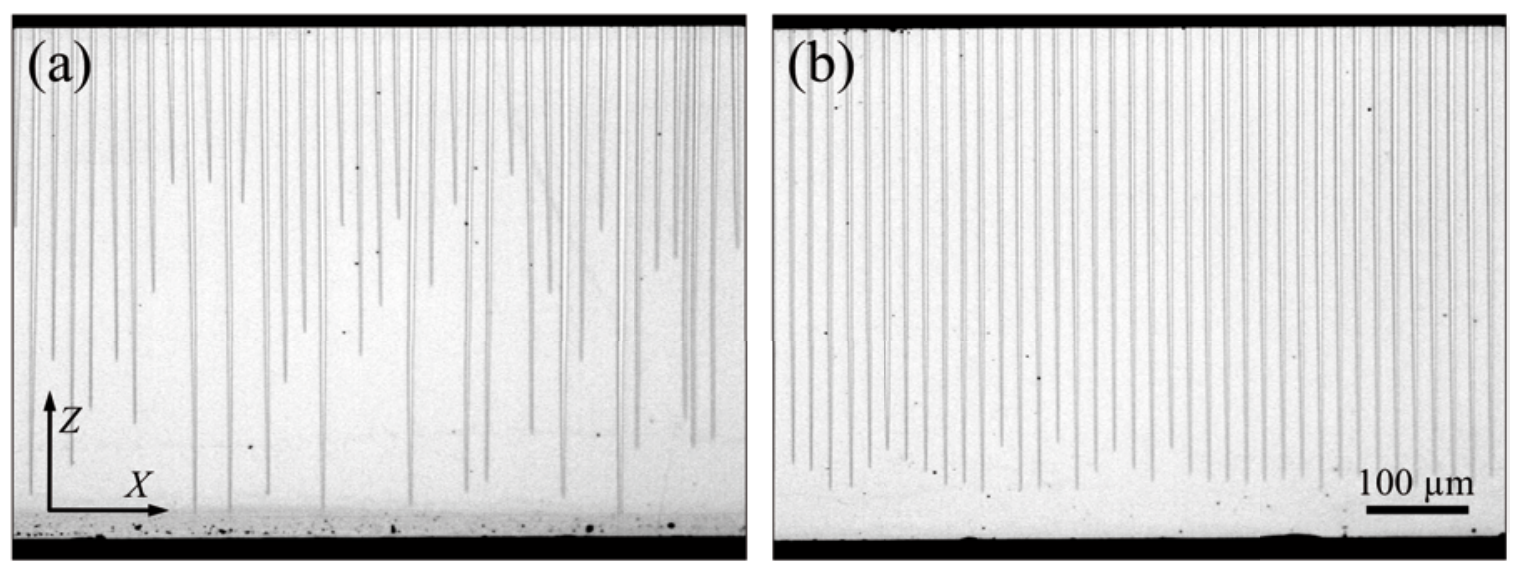

FIG. 2. Optical microscope images of the HF-etched cross-sections of the crystal after (a) the first poling cycle and (b) the second poling cycle. The periodic, needle like structure 
are the inverted domains (correspond to the bulk domain in Fig. 1b), which start at the $+Z$ face and become thinner as they grow in depth. The domain depth uniformity improves after the second poling cycle.
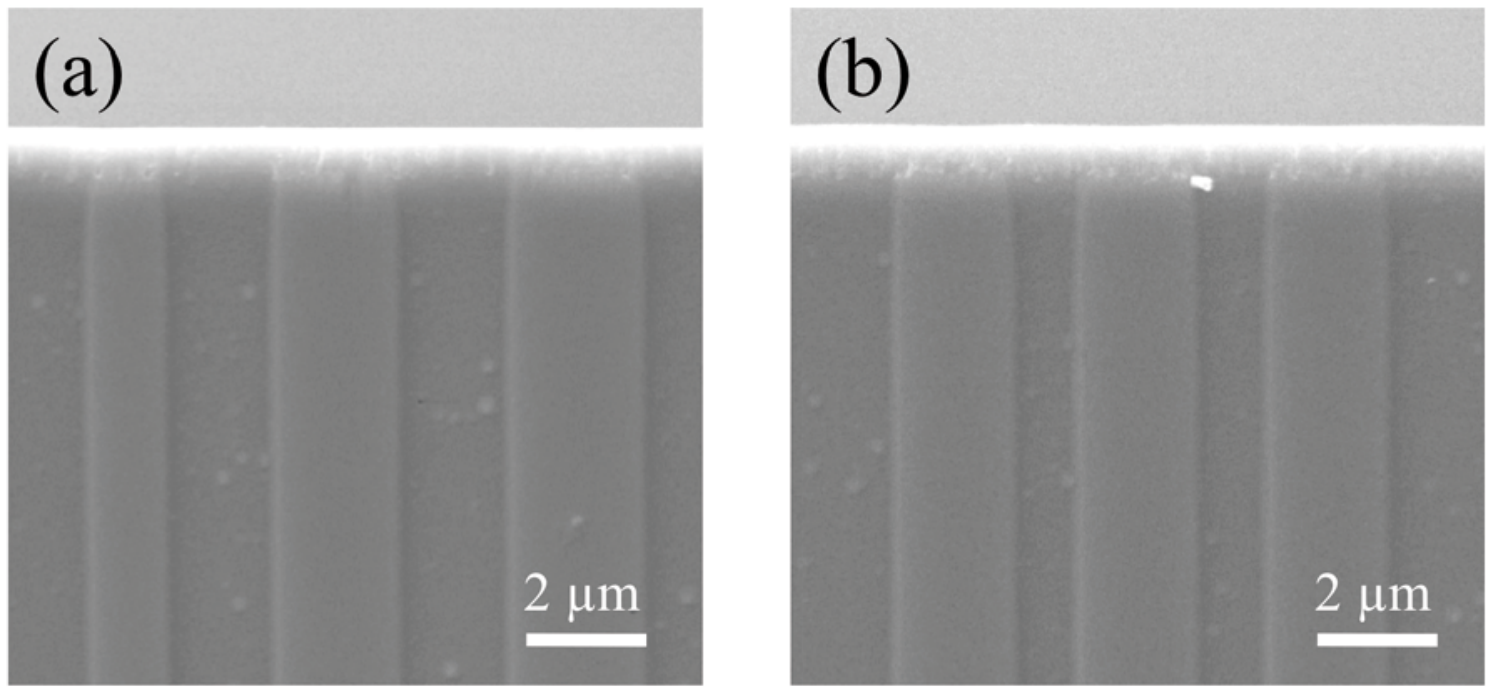

FIG. 3. SEM images of the HF-etched cross-sections for a scanning period of (a) $4 \mu \mathrm{m}$ and (b) $3 \mu \mathrm{m}$. 


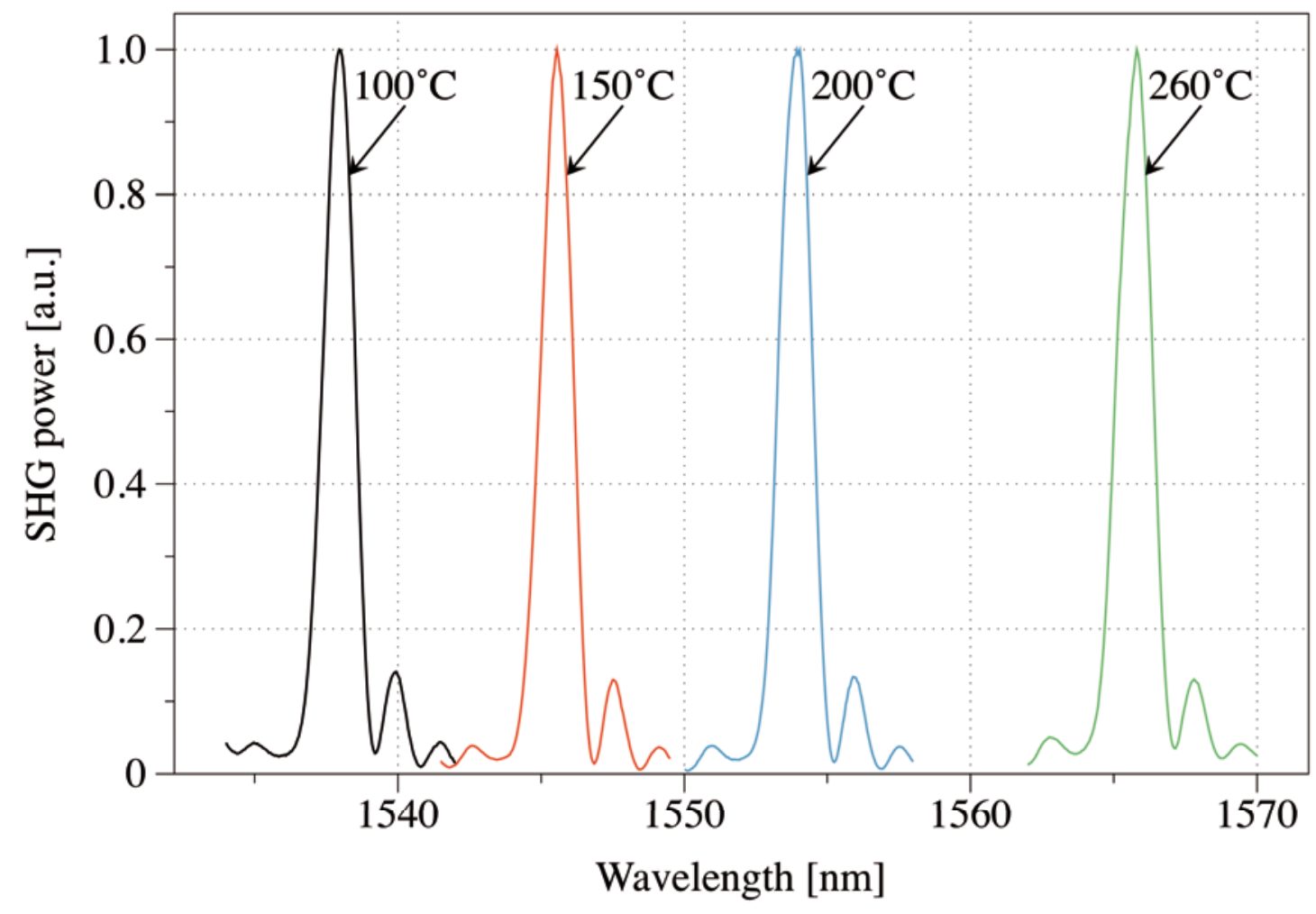

Fig. 4: Normalized SHG power as a function of the fundamental wavelength for a temperature of $100,150,200$ and $260^{\circ} \mathrm{C}$. 


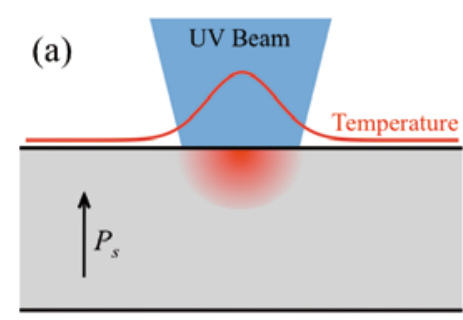

(d)

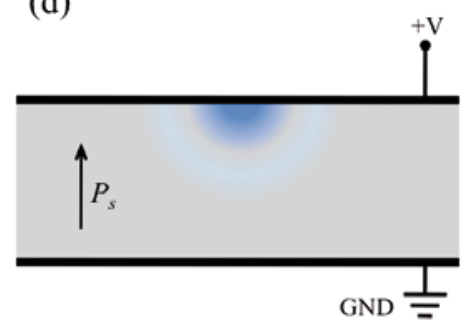

(g)

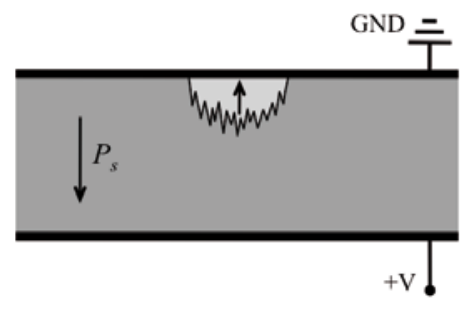

(b)

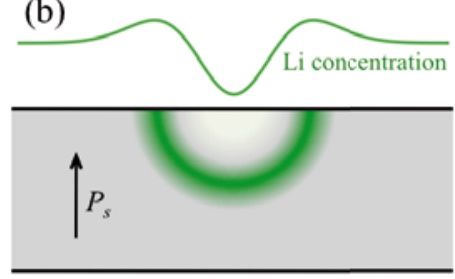

(e)

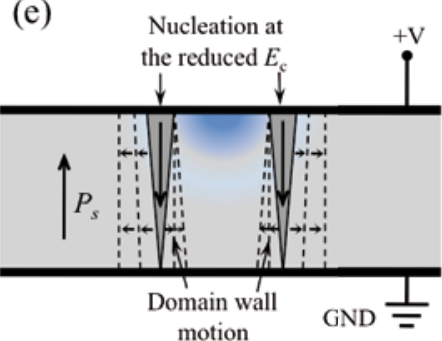

(h)

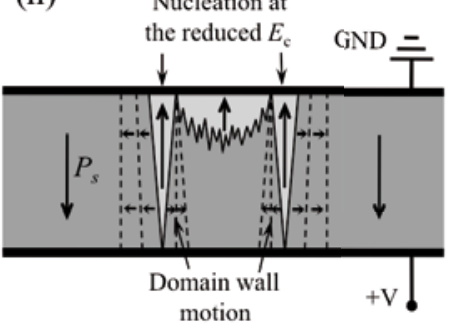

(c)

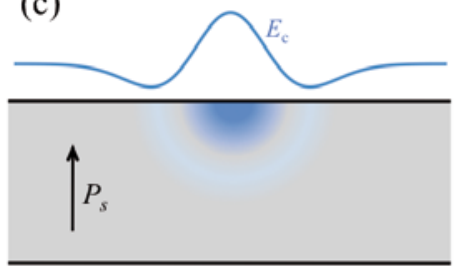

(f)

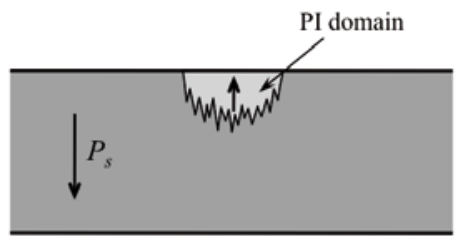

(i)

Polarization underneath PI domain is maintained

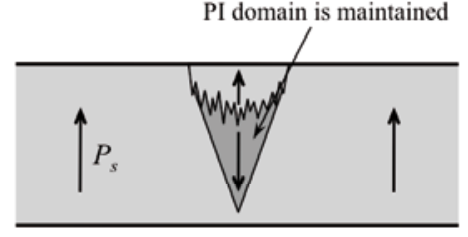

FIG. 5. Schematic illustration of the proposed domain inversion process for generating bulk domains that were defined by UV laser light irradiation. The focused UV light is strongly absorbed at the crystal surface, generating a local heat profile (a), which causes Li diffusion into the colder adjacent crystal (b). The $E_{\mathrm{c}}$ depends on the Li concentration (c). When a forward poling step is applied (d), the domains prefer to nucleate at the $\mathrm{Li}$ enriched region next to the UV-irradiated track (e). The Li deficient region on the other hand is poling inhibited, resulting in a surface domain when the poling step is completed (f). When applying the reverse poling step (g), again the domains prefer to nucleate at the Li enriched region (h). However, this time the area below the PI domain maintains its polarization, forming a bulk domain (i). 